\title{
El reto de formar a periodistas participativos: reflexiones docentes ${ }^{1}$
}

\author{
Bella Palomo \\ Universidad de Málaga \\ bellapalomo@uma.es \\ María SÁNCHEZ \\ Universidad de Málaga \\ m.sanchezgonzalez@uma.es
}

\begin{abstract}
Resumen:
Las audiencias activas y la industria mediática están modificando el modelo tradicional de graduado en Periodismo, demandando jóvenes multitarea activos en las plataformas sociales. Partiendo de dicha premisa, esta investigación analiza la implantación de los nuevos planes de estudio en la universidad española en la era de los medios sociales y recopila las reflexiones de sus protagonistas, mediante entrevistas en profundidad con doce profesores responsables de asignaturas vinculadas directamente con el periodismo participativo.
\end{abstract}

Palabras clave: periodismo participativo; interactividad; redes sociales; universidad

\section{The challenge of training participatory journalists: faculty perceptions}

\begin{abstract}
:
Active audiences and media industry are changing the traditional model of journalism graduate, demanding multi-skilled and social media-savvy young journalism graduates. Based on this premise, this research analyzes the implementation of new curricula in Spanish university in the age of social media and collects the reflections of its protagonists. 12 in-depth interviews were conducted with faculty teaching participatory journalism.
\end{abstract}

Key Words: participatory journalism; interactivity; social networks; university

Referencia normalizada:

Palomo, B. y Sánchez, M. (2014): El reto de formar a periodistas participativos: reflexiones docentes. Historia y Comunicación Social. Vol. 19. Núm. Especial Febrero. Págs. 465-478.

Sumario: 1. Introduccion. 2. Se busca gestor de comunidades. 3. Nativos digitales vs. inmigrantes digitales. 4. Metodología. 5. Resultados. 5.1. Una asignatura anti-crisis. 5.2. Atrapados por la ingenuidad. 5.3. El nuevo lado oscuro del periodismo. 5.4. To tweet or not to tweet. 5.5. Éxito y futuro. 6. Conclusiones. 7. Referencias bibliográficas

1 Esta investigación se ha desarrollado en el marco del proyecto de investigación nacional "Audiencias Activas y Periodismo: Estrategias de Innovación para la Empresa Informativa y Nuevas Figuras Profesionales", con código CSO2012-39518-C04-04, financiado por el Ministerio de Economía y Competitividad. 


\section{Introducción}

Tradicionalmente, la dinámica del sector de la comunicación ha influido en la formación de los periodistas, lo que justificó ya en 1969 el lanzamiento de la revista científica Journalism \& Mass Communication Educator y la aparición regular de artículos científicos que abordan la cuestión (Deuze, 2001). La academia, por tanto, siempre ha seguido de cerca las tendencias de la industria. Como muestra de ello, las relaciones multimedia o los casos de convergencia se han abordado con interés en asignaturas vinculadas con la estructura del sistema de medios; la nueva maquetación de un periódico centraba alguna clase de diseño, y los contenidos han permitido analizar la actualidad nacional e internacional según la agenda de los medios. Por ello no resulta sorprendente que el surgimiento de las audiencias activas, que ha modificado el consumo informativo y las rutinas profesionales, haya alterado también los planes de estudios en las facultades de Comunicación.

Este artículo analiza si las universidades españolas públicas y privadas han orientado sus planes de estudios a las nuevas exigencias del mercado, descubre bajo qué formulas se está integrando el periodismo participativo en la docencia, y cuáles son las ventajas y los inconvenientes que acompañan a su enseñanza. Se trata de un estudio útil, actual y, sobre todo, necesario porque atravesamos una época de transición en la que incluso algunos docentes dudan de la necesidad futura de los estudios de Periodismo (Claussen, 2012). Por ello, actualmente, el gran reto del profesorado de Periodismo es continuar siendo relevante en un entorno informativo y educativo tan cambiante.

\section{Se busca gestor de comunidades}

La drástica reducción de la difusión de los medios tradicionales, la pérdida de anunciantes y el recorte de las redacciones han incrementado la dependencia entre las empresas periodísticas y sus audiencias. En esta nueva etapa, el periodista no solo se responsabiliza de la producción de los contenidos; también se encarga de la distribución, promocionando informaciones del medio, y del cliente, estableciendo sinergias con los públicos con la aspiración de fidelizarlos. Mostrar en qué estás trabajando, cómo lo haces y estar abierto a preguntas y sugerencias puede hacer sentir a los lectores que realmente el medio está actuando teniendo en cuenta sus intereses.

Los periodistas y los ciudadanos están más próximos que nunca, y hay datos significativos que constatan la magnitud de estos nuevos hábitos: El 89\% de los periodistas estadounidenses afirman que recurren a blogs para completar sus investigaciones; el $65 \%$ a redes sociales como LinkedIn y Facebook; el 58\% utiliza espacios donde se comparten imágenes y vídeos; un $52 \%$ prefiere Twitter y un $42 \%$ acude a foros de debate (Social Media \& Online Usage, 2009). Las cifras nacionales tampoco resultan despreciables: En octubre de 2013, El Mundo mantenía 260 cuentas en Twitter, 
(a)el_pais arrastraba a más de tres millones de seguidores y su producción de tuits (118.796) superaba al mismísimo@nytimes (116.964). Además, la periodista de Cuatro Carmela Ríos, fue galardonada en los Premios Ortega y Gasset de Periodismo 2012 por la cobertura realizada del 15M a través de Twitter.

Al margen de la actividad que les une en las redes sociales, se emplean también nuevas herramientas, como CoveritLive, que favorecen una conexión permanente con los públicos basadas en el liveblogging, se admite la colaboración amateur en la producción informativa e incluso Wikipedia ha empezado a ganarse el respeto de los periodistas hasta el punto de influir en las agendas mediáticas (Messner y South, 2010: 153).

Esta intensa actividad exige una reflexión sobre el nivel de adaptación de los planes de estudios a este escenario. ¿Están capacitados los nuevos egresados para gestionar comunidades? ¿Cuál es la formula idónea para preparar en la Universidad a las futuras generaciones de periodistas? La respuesta es compleja, ya que la inestabilidad y el futuro incierto del sistema mediático impiden diseñar planes de estudio vigentes a medio plazo, lo que simultáneamente acentúa la desconexión entre la academia y la empresa (Du y Thornburg, 2011). Sí parece existir acuerdo en implantar itinerarios de formación basados en la convergencia, en una flexibilidad multiplataforma que diseñe periodistas polivalentes (Micó, Masip y Ballano, 2012). El modelo de enseñanza aislado, cuya estructura separa la prensa, la radio, la televisión e internet, no es viable (Hirst y Treadwell, 2011: 450) y esta adaptación resulta clave porque el mercado laboral actual demanda periodistas multitarea que además sean expertos en medios sociales, y la universidad no puede ignorar esta realidad. Muchas de las nuevas salidas laborales (community manager, SEO) dependen de su iniciativa emprendedora y su esfuerzo como autónomos. De hecho, según datos de la Asociación de Trabajadores Autónomos, en España hay 48.460 freelance y autónomos del periodismo. Durante la crisis, éste ha sido el único sector en alza entre los autónomos, dándose de alta 2.094 profesionales, y se estima que esta atomización y reactivación de la profesión es consecuencia de la expansión de la comunicación digital en su vertiente 2.0 (Mandel, 2013).

\section{Nativos digitales vs. inmigrantes digitales}

Uno de los handicaps para la implantación del cambio es que mientras los estudiantes son nativos digitales, la mayoría de los profesores son inmigrantes digitales (Prensky, 2001). Existen esfuerzos desde la academia para eliminar esta brecha, planteando cursos específicos de innovación educativa o desarrollando el perfil del 'open scholar' (Pearce, Weller, Scanlon y Ashleigh, 2010), es decir, aquel docente que toma la iniciativa de publicar sus contenidos en abierto, vía blogs o como los vídeos elaborados por Michael Wesch, profesor de la Universidad Estatal de Kansas, para su proyecto Mediated Cultures, que en octubre de 2013 se acercaban a los 22 
millones de reproducciones en Youtube, una cifra que excede con creces la audiencia que se puede alcanzar en libros o revistas científicas.

Geográficamente, esta inquietud no entiende de fronteras, y se han realizado aproximaciones al fenómeno en lugares tan dispares como Nueva Zelanda, Estados Unidos, Reino Unido, Finlandia o España. Estos estudios se centran fundamentalmente en ofrecer recomendaciones para que las facultades se adapten al nuevo escenario (Murray, 2008; Molina, 2008); describen cómo se integran los nuevos perfiles profesionales en el aula (Núñez, Teixeira y García, 2012); estudian la inclusion curricular del periodismo on-line (Tejedor, 2008); monitorizan el uso de Facebook y Twitter como herramientas docentes (García, de la Morena y Melendo, 2012); analizan los conocimientos técnicos de los estudiantes (Hirst y Treadwell, 2011); recopilan qué competencias demanda la industria en las nuevas contrataciones, qué expectativas tienen de los egresados y qué debilidades detectan en las nuevas generaciones de periodistas (Brown y Collins, 2010); analizan las ofertas de empleo que buscan profesionales 2.0 (Wenger y Owens, 2012), la experiencia previa requerida, y lo contrastan con los cursos de posgrado que ofrecen las universidades (Sánchez y Méndez, 2013), o incluso proponen fórmulas para implantar la innovación en la formación del periodista (Lassila-Merisalo y Uskali, 2011).

Entre otras cuestiones, estos análisis apuntan que a pesar del compromiso que tienen los jóvenes con las redes sociales, todavía no se sienten del todo cómodos aplicando estas técnicas en el terreno profesional al crear, por ejemplo, contenidos para un blog o publicando un vídeo en Youtube (Hirst y Treadwell, 2011). El estudiante de Periodismo se identifica más con el consumidor que con el productor; su actitud pasiva, receptora, prevalece sobre la oportunidad proactiva del nuevo ecosistema comunicacional, y sus audiencias se reducen a la familia y a los amigos, lo que evidencia la brecha entre las prácticas profesionales y las de los alumnos de Periodismo.

Otro de los pilares fundamentales, la empresa, considera que la formación debe desarrollarse sobre tres ideas: el buen periodismo no tiene fecha de caducidad; el buen periodismo funciona en todos los soportes, y la flexibilidad es una exigencia para sobrevivir en el escenario presente y futuro (Finucane, 2006).

Desde el punto de vista formativo, existen investigaciones que analizan el uso académico que hacen los universitarios de las redes sociales (Gómez, Roses y Farias, 2012), pero no especifican cómo se forma a los futuros periodistas en esta práctica. Este curso 2013/2014 se completa la implantación del grado en todas las facultades españolas, y es por tanto el momento idóneo para hacer balance sobre las novedades implementadas en materia de interacción con las audiencias. 


\section{Metodología}

Las metodologías con las que se ha abordado el impacto que las nuevas rutinas profesionales poseen en la formación de las nuevas generaciones de periodistas son básicamente cuatro: el análisis de contenido aplicado a las guías docentes y las ofertas de empleo; los estudios descriptivos de casos, que fundamentalmente narran la experiencia propia de los investigadores; las entrevistas en profundidad, y las encuestas. Esta última técnica es la más extendida, y se ha aplicado para conocer el uso que los estudiantes otorgan a los medios sociales, por qué abren una cuenta y cómo valoran estas herramientas en el contexto profesional, la disonancia existente entre los conocimientos de los periodistas y los estudiantes, o para que los responsables de los medios determinen las habilidades multimedia que exigen en las nuevas contrataciones.

La presente investigación, de carácter cualitativo, ha recogido la información aplicando la técnica de la entrevista en profundidad no estructurada, con objeto de recolectar datos, opiniones y experiencias de profesores responsables de asignaturas vinculadas directamente con el periodismo participativo, y describir el contexto en el que se desarrollan. Debido a la reciente implantación del grado de Periodismo en España, estas entrevistas poseen también un carácter exploratorio, ya que ofrecen la oportunidad de elaborar un marco de actuaciones futuras.

En una primera fase se analizaron los planes de estudios de los grados de Periodismo ofertados en 33 universidades públicas y privadas españolas para el curso 2013/2014, con objeto de detectar los centros donde se daba un mayor protagonismo al periodismo participativo. Quedaron excluidos del estudio aquellas universidades cuyas programaciones no estaban disponibles o detalladas en internet. Se detectaron 22 asignaturas que cumplían con el criterio de que más del $50 \%$ de su programa estaba relacionado con la interacción con las audiencias. Se remitió por correo electrónico una solicitud de entrevista para colaborar en el estudio a todos los profesores responsables de dicha docencia, y contestaron positivamente doce: Cristina Aced (Universitat Abat Oliba), Montecarlo Blanquerna (Universitat Ramón Llull), María Ganzábal (Universidad del País Vasco), Elvira García de Torres (Universidad Cardenal Herrera), Carmen Haro (Universidad de Valladolid), David Herrera (Universidad a Distancia de Madrid), Xosé López (Universidad de Santiago de Compostela), José Luis Orihuela (Universidad de Navarra), Karma Peiró (Universitat Ramón Llull), Teresa Sandoval (Universidad Carlos III), Nuria Simelio (Universidad Autónoma de Barcelona) y Bárbara Yuste (Universidad Camilo José Cela). Dichos encuentros fueron telefónicos o vía Skype, se celebraron en septiembre y octubre de 2013, y duraron entre 40 y 60 minutos. 


\section{Resultados}

Las habilidades tecnológicas han pasado de ser un añadido opcional a constituir el eje central de los estudios de Comunicación. Algunas facultades han creado incluso itinerarios específicos en ciberperiodismo.

En España se han localizado veinte universidades que imparten en su conjunto 22 asignaturas directamente relacionadas con el periodismo participativo (ver tabla 1). El 59\% de ellas tienen carácter obligatorio o de formación básica en los planes de estudios, por lo que esta introducción del periodismo abierto en el sistema curricular posee una consideración mayor a la que en su momento tuvo el ciberperiodismo en los planes de estudio, que fue considerada una especialización fundamentalmente opcional. Los alumnos son conscientes de la relevancia que este tipo de contenidos posee para su formación. Según una encuesta realizada en el curso 2012/2013 por la profesora Elvira García de Torres a sus alumnos de la asignatura obligatoria Comunicación Digital y Participación Ciudadana en la Red, el 90\% se habría matriculado de ella si fuera optativa.

Esta oferta formativa se realiza especialmente durante los últimos dos cursos del grado. Solo las Universidades de Navarra, Carlos III y Ramón Llull introducen estas cuestiones el primer año de carrera. Teresa Sandoval es responsable de una de ellas, Comunicación y Participación Ciudadana en la Red: Para la Carlos III "era fundamental incluir en $1 .^{\circ}$ una asignatura que conectara con el alumnado, que fuera moderna y actual... Continúa la línea de Teoría de la Comunicación junto a una dimensión práctica previa a Periodismo en la Red". Esta asignatura, que se puede cursar también en inglés, ha sido además pionera en España en abordar el periodismo participativo, ya que se lleva impartiendo cinco cursos académicos. Para elaborar sus contenidos, su referencia fundamental fue Estados Unidos.

En este momento de transición y asentamiento de nuevas asignaturas que nacen respaldadas por la web 2.0, los profesores aprenden tanto de sus alumnos como estos de sus profesores. Descubrir, integrar, aplicar y enseñar estos entornos son las funciones básicas del docente especializado en periodismo participativo. 
Tabla 1. El periodismo participativo como asignatura autónoma en los grados de Periodismo en España (curso 2013/2014)

IE University*

Universidad a Distancia de Madrid Universidad Autónoma de Barcelona Universidad Camilo José Cela Universidad Camilo José Cela Universidad Carlos III

Universidad CEU Cardenal Herrera Universidad de Castilla-La Mancha Universidad de Deusto*

Universitat de Lleida

Universidad de Málaga

Universidad de Navarra

Universidad de Santiago de Compostela

Universidad de Valladolid

Universidad del País Vasco

Universidad Francisco de Vitoria

Universidad Internacional de La Rioja*

Universitat Abat Oliba CEU

Universitat de les Illes Balears

Universitat de Vic

Universitat Ramón Llull

Universitat Ramón Llull
Comunidades virtuales

Periodismo digital y redes sociales

Producció periodística multiplataforma

Nuevas tecnologías

Redes sociales

Comunicación y participación ciudadana en la red

Comunicación digital y arquitectura de la información

Comunicación interactiva y redes sociales

Elaboración de mensajes para la web

Gestión y producción de la información en red

Periodismo ciudadano y redes sociales

Comunicación multimedia

Movimientos periodísticos y comunicación ciudadana

Periodismo participativo en la red

Periodismo social y participativo en internet

Ciberperiodismo y productos digitales

Comunicación en medios digitales

Producció periodística

Community management

Mitjans de comunicació socials

Periodismo digital

Narrativa multimedia

\begin{tabular}{|c|c|c|}
\hline Obligatoria & 3 & 3.9 \\
\hline Formación básica & 6 & 4.음 \\
\hline Optativa & 6 & 4.음 \\
\hline Obligatoria & 6 & 2.0 \\
\hline Optativa & 6 & 3.o \\
\hline Formación básica & 6 & $1 . \mathrm{o}$ \\
\hline Obligatoria & 6 & 4.9 \\
\hline Optativa & 6 & 4.9 \\
\hline Obligatoria & 6 & 3.9 \\
\hline Obligatoria & 7,5 & $3 . \mathrm{o}$ \\
\hline Optativa & 6 & 3.o-4.o \\
\hline Obligatoria & 3 & 1.0 \\
\hline Optativa & 6 & 4.음 \\
\hline Optativa & 7,5 & 3.o \\
\hline Optativa & 6 & $3 . \mathrm{o}$ \\
\hline Obligatoria & 6 & 3.9 \\
\hline Obligatoria & 4 & 3.9 \\
\hline Obligatoria & 6 & 3. 9 \\
\hline Obligatoria & 6 & 4. $\mathrm{o}$ \\
\hline Optativa & 3 & \\
\hline Obligatoria & 6 & 4.9 \\
\hline Optativa & 2 & 1.0 \\
\hline
\end{tabular}

*Con asterisco los grados en Comunicación

\subsection{Una asignatura anti-crisis}

Durante décadas periodistas y docentes han ignorado las señales de alarma que avisaban de la posible extinction de los periódicos. Pero en la actualidad, muchos profesores son conscientes de que están formando a expertos en comunicación para que asuman sin traumas futuros trabajos que, en algunos casos, todavía no han sido inventados. Esta actitud abierta caracteriza a los profesores que imparten asignaturas vinculadas con la interacción con las audiencias, y proyecta el interés común de prolongar la vida útil la profesión periodística. Las redes sociales tienen un gran protagonismo en esta nueva reconversión profesional. Para Bárbara Yuste, docente en la Universidad Camilo José Cela, "Twitter o Facebook pueden desaparecer pero no la forma en la que ya se relacionan periodistas y usuarios. Estoy convencida de que no se puede retroceder".

Todos los docentes entrevistados consideran que los responsables de impartir asignaturas vinculadas con el periodismo digital y el periodismo participativo deben responder a un perfil específico, que aporten conocimientos sólidos de teoría, experiencia en la utilización de medios sociales y que estén familiarizado con la dinámica de trabajo en entornos 2.0. Este contexto requiere una evaluación permanente del programa, una exploración constante de los nuevos hábitos comunicacionales $\mathrm{y}$, por lo tanto, una mayor exigencia para el profesor porque no es una asignatura cómoda. Los profesionales en activo que la imparten cuentan con la ventaja de trasladar al alumno un amplio número de experiencias reales, al igual que aquellos que pert- 
enecen a proyectos de investigación consolidados vinculados a la innovación en comunicación, aunque todos se consideran autodidactas en estas cuestiones.

Las entrevistas desvelan que la mayoría de los profesores son conscientes de que esta oferta formativa no es homogénea en todas las universidades; saben que ha existido una resistencia académica, similar a la de la industria mediática, hacia internet, y la disrupción cultural que han provocado los nuevos medios les ha pillado a algunos por sorpresa. Narrar la evolución de los modelos resulta lógico y coherente, pero las actitudes de rechazo, ataque o incluso desprecio a las asignaturas vinculadas con los nuevos medios ya no tienen sentido porque el futuro es digital (Nosty, 2013) y están dañando a la profesión si los egresados no salen con la preparación necesaria para afrontar los nuevos retos del mundo laboral. Además, como explica Carlo Blanquerna, "contar que el periodismo está en crisis no atrae nuevos alumnos; las nuevas salidas, sí". Así, profesores que durante años han sido considerados frikis ahora encajan con la normalidad del funcionamiento de las empresas. Aunque la crítica también salpica al sector. Como indica Bárbara Yuste, a veces "las propias empresas no le dan la importancia que tiene, y contratan un perfil junior para mantener sus cuentas en las redes sociales".

\subsection{Atrapados por la ingenuidad}

En cualquier caso, no sacrifican las bases del periodismo pero sí enseñan a los jóvenes aspirantes a periodistas a comprender el mundo digital. El equilibrio entre teoría y práctica resulta fundamental porque los alumnos necesitan conocer tanto las aplicaciones como los argumentos para decidir su adecuado uso. En este sentido, a los profesores no les preocupa que algún alumno pueda superarles en conocimientos técnicos porque, aunque están muy familiarizados con las herramientas, el uso que le otorgan no es profesional aunque creen que por tener 500 amigos en Facebook ya son expertos, y consideran que el tono y el lenguaje profesional en estos entornos es el mismo que el que usan con sus amigos. Éste es un objetivo docente común: transformar al nativo digital en un profesional con destreza digital. José Luis Orihuela, que lleva desde finales del siglo XX trabajando con nuevos medios y es autor del blog de referencia eCuaderno, ha detectado también este uso inocente de las redes en sus alumnos de Comunicación Multimedia: "Hay una destreza generacional orientada a mantener relaciones a distancia con grupos primarios, no para generar valor, o incluso negocio". Detectar esta carencia le ha permitido cambiar la mentalidad del alumno y guiarlo hacia el trabajo autónomo y el emprendimiento, reto que aceptan porque "son conscientes del terrible momento que atraviesa el sistema, lo que ha reforzado el carácter vocacional de la profesión”, puntualiza el profesor de la Universidad de Navarra.

Esta ingenuidad la detectan también en las redacciones. Aquellos docentes que han ejercido o siguen ejerciendo la profesión reconocen que cuando llegan becarios hay que educarlos bastante, no tanto en cuestiones técnicas, como en los valores fundacionales del periodismo. 
Montecarlo Blanquerna afirma que los nativos digitales son una leyenda urbana: "No hay un único perfil de alumno y en clase apenas tengo tres videojugadores; algunos ni siquiera están en Facebook". A pesar de ello, sus alumnos de una asignatura muy específica que imparte en $1 .^{\circ}$ sobre narrativa transmedia hacen networking desde el primer día de clase. Las claves de este compromiso: la adaptación pedagógica y su dimensión profesional ya que "a los chicos les da seguridad y el profesor adquiere credibilidad si fuera del aula detectan que sabes y practicas lo que explicas", comenta el profesor de la Ramón Llull. A veces les amenaza con dar la clase por Facebook, y siempre usa "un discurso muy fragmentado, muy similar a como son los medios hoy".

\subsection{El nuevo lado oscuro del periodismo}

La visión tradicional que los estudiantes siguen teniendo de la profesión es otro hándicap que puede retrasar su compromiso con la asignatura. A pesar de su intensa actividad personal en las redes sociales, pocos alumnos se plantean una salida profesional relacionada con los medios sociales. Según Cristina Aced, de la Universitat Abad Oliva, "antes el lado oscuro era trabajar en gabinetes de prensa; ahora esto es lo que consideran de segunda categoría". Nuria Simelio piensa que el motivo es cultural, "les da miedo buscarse la vida", y no desconocimiento porque "desde $1 .^{\circ}$ se les habla bastante de estas nuevas profesiones", y en la Autónoma llevan invitados que viven del microperiodismo en internet. Carmen Haro también ha observado esta actitud en sus alumnos de Valladolid: "Han hecho terapia de grupo porque tienen una visión muy negativa de su futuro ya que han empezado a estudiar en plena crisis", y por ello cree que su oportunidad consiste en centrarse en sus valores: "son más generosos, tienen más clara la idea del intercambio y la comunidad; todo lo ponen en común".

Esta mirada conservadora afecta también al conocimiento que tienen de la participación ciudadana. Para Karma Peiró, vinculada profesionalmente durante dos décadas a redacciones digitales catalanas y responsable de Periodismo Digital en la Universitat Ramón Llull, "trabajar con audiencias anónimas implica un gran respeto, tacto y una verificación obligatoria. Sin embargo, la visión de los estudiantes es que la red da poder a la gente para que informen; proyectos de periodismo ciudadano los ven como un intrusismo a la profesión, lo cual es una vision simplista y conservadora de qué significa ser periodista". David Herrera, community manager en IBM España y profesor de Periodismo Digital y Redes Sociales en la Universidad a Distancia de Madrid, ofrece a sus alumnos varios consejos para sobrevivir en estos entornos: "Hay que seguir a los diez periodistas de más éxito en Twitter, blogs, que no teman descargarse aplicaciones y que sigan a las máximas referencias en estos temas".

Algunas de las asignaturas vinculadas con el periodismo participativo han expandido su función utilitaria transformándose en incubadoras de innovación (Lynch, 2007), que ayudan a alumnos emprendedores a experimentar y empezar a definir una estrategia profesional futura. Para ello proponen crear productos que superen los 
límites físicos del aula. Las publicaciones digitales Off Topic, Periodistillas, Valladolid a tu Alcance o dorsal51 son ejemplos de estas iniciativas.

\subsection{To tweet or not to tweet}

Los medios sociales han revolucionado las aulas. No solo se integran con un uso pedagógico o representan un objeto de estudio, sino que también muchos alumnos asisten a clase con sus perfiles abiertos. Ante esta conexión permanente con las redes sociales, la respuesta docente es muy heterogénea. Hay quien solo prohíbe el uso de las redes en las clases teóricas y permite que en las prácticas las usen como canal de información de actualización constante; algunos asumen que van a competir con Facebook y eso les motiva a dar las clases de una forma más atractiva para captar su atención; otros docentes consideran que limitar estos usos contradice el espíritu de la propia asignatura y animan a que se tuiteen sus clases tomando notas y publicándolas en tiempo real. En este sentido, no pueden ni quieren evitarlo porque saben que esta nueva generación de alumnos tiene la capacidad de hacer varias cosas simultáneamente.

Otro de los temores que abordan a los docentes es el riesgo de plagio que acompaña a estas asignaturas. Muchos alumnos empezaron a copiar contenidos de Wikipedia durante su etapa en el instituto. Esto ha influido en el escaso valor que otorgan a las fuentes, lo que queda patente cuando, por ejemplo, elaboran un glosario. Los docentes se centran en reeducarlos para que superen estos vicios. En los casos estudiados, el plagio es motivo de suspenso y para evitar la tentación algunos profesores plantean ejercicios que no puedan copiarse, basados en comparaciones.

\section{5. Éxito y futuro}

El diseño de este tipo de asignaturas, basado en una intensa evaluación continua donde un alto porcentaje de la nota se basa en el trabajo del estudiante, permite que el porcentaje de aprobados sea muy alto, superando en la mayoría de los casos el $90 \%$. Estos datos y el empleo de herramientas sociales favorecen que haya menos frustración que en otras asignaturas. Una de las quejas más extendidas entre los estudiantes es precisamente el número de prácticas que deben asumir, ya que en algunos casos oscila entre 16 y 20 ejercicios. El mantenimiento de un blog es la actividad más usual, y las temáticas preferidas por los estudiantes son la moda y los deportes. Una segunda queja estaría relacionada con recibir estos contenidos el ultimo trimestre de la carrera, porque entienden que influye en la concepción de la profesión y deberían conocer estas nuevas oportunidades mucho antes de concluir el título.

La queja más extendida por parte del docente es que muchos alumnos se confían al ver que la asignatura aborda las redes sociales, y creen que va a ser un puro trámite. Esto ha provocado en alguna ocasión tener que reconfigurar el temario para tratar cuestiones que desconocen, como el periodismo de datos, el SEO aplicado a la redacción, la brecha digital, los nuevos perfiles, el posicionamiento, el crowdfunding, la filosofía del procomún, la ética hacker o forjar su marca personal. 
Aunque la mitad de los entrevistados perciben que otras asignaturas invaden su contenido, en este sentido, hay una sugerencia de mejora muy extendida en el panel de expertos empleado: incrementar el número de materias que traten lo digital de forma transversal para fomentar la producción transmedia. Como indica Xosé López, catedrático de Periodismo y responsable de Movimientos Periodísticos y Participación Ciudadana en la Universidad de Santiago, "estamos al principio de un proceso de cambio, con modificaciones periódicas en las materias". Las asignaturas vinculadas a la comunicación participativa son las más innovadoras que podemos encontrar en un plan de estudios en la actualidad, aunque el periodismo de datos y los estudios prospectivos pueden tomar la alternativa en las próximas revisiones.

\section{Conclusiones}

La industria mediática transforma continuamente las nuevas generaciones de periodistas. En una primera fase, las habilidades multimedia resultaron imprescindibles para que los periodistas poseyeran un perfil competitivo. En la actualidad, la dimensión participativa del comunicador es el gran valor en alza. Por ello ni la academia ni las empresas pueden ignorar la importancia de los medios sociales, el contenido generado por la audiencia y cómo afectan estos a las rutinas profesionales. Un alumno adaptado a esta realidad debe preguntarse cuestiones del tipo: ¿Cómo puedo atraer la atención de la audiencia? ¿Cómo conseguir que una noticia sea popular y fluya del medio a Facebook o Twitter? ¿Puedo construir contenidos interactivos para cualquier tipo de pantalla y audiencia? ¿Cuáles son los límites que acompañan a esta actividad transmedia?

La mitad de los grados de Periodismo que se imparten en España incluyen en sus planes de estudios asignaturas vinculadas al periodismo participativo que pueden dar respuesta a estas cuestiones. Hablar de redes sociales en el aula se ha normalizado.

La principal complejidad detectada en este tipo de asignaturas procede del heterogéneo perfil tecnológico del alumno (tecnófobos, escépticos, usuarios enganchados a las redes...) y de su elevado interés por las salidas profesionales convencionales. Actualmente no resulta sorprendente que la mayoría de los alumnos sean activos en las redes sociales, ya que muchos de ellos poseen portátiles, tabletas y smartphones y acuden con ellos al aula. Lo peculiar es que confunden el conocimiento de usuario con la aplicación profesional, y las preferencias tradicionales de los alumnos como futuros profesionales productores de contenidos no coinciden con las de la audiencia, más próximas al consumo de información en soportes móviles y a través de redes sociales.

Los estudios de Periodismo atraviesan el mayor reto pedagógico desde su existencia: formar a periodistas en un escenario marcado por la crisis, la incertidumbre y los cambios impredecibles. La crisis del modelo de negocio obliga a reinventarse y superar muchas de las prácticas del pasado. A pesar de ello, se detecta una brecha 
digital en el seno de la Universidad, donde profesores innovadores conviven con otros docentes que se resisten a reducir el protagonismo que han otorgado a los dinosaurios mediáticos en sus programaciones.

En este periodo de transición, la convivencia entre el modelo actual y el tradicional requiere de una gran coordinación docente que evite solapamientos y ausencias en los contenidos de los temarios y, sobre todo, sentar las bases de una nueva cultura formativa basada en un constante aprendizaje del docente, al igual que le ocurre al periodista.

\section{Bibliografía}

BROWN, T.; COLLINS, S. (2010). "What "they" want from "us": Industry expectations of journalism graduates". En: Electronic News, vol. 4, n. ${ }^{\circ}$ 2, p. 68-82.

CLAUSSEN, D. (2012). "If even journalism professors don't know what journalism is, then all really is lost". En: Journalism \& Mass Communication Educator, vol. 67, n. ${ }^{\circ}$, p. $327-331$.

DEUZE, M. (2001). "Educating 'new' journalists: challenges to the curriculum". En: Journalism \& Mass Communication Educator, vol. 56, n. ${ }^{\circ} 1$, p. 4-17.

DÍAZ NOSTY, B. (2013). La prensa en el nuevo ecosistema informativo. "iQue paren las rotativas!» Barcelona: Ariel. Fundación Telefónica.

DU, Y.R.; THORNBURG, R. (2011). "The gap between online journalism education and practice: the twin surveys". Journalism \& Mass Communication Educator, vol. $66,{ }^{\circ}{ }^{\circ}$, p. $218-230$.

FINUCANE, P. (2006). "Teaching Journalism for an unknown future". Nieman Reports, vol. 60, n. ${ }^{\circ} 1 .<$ http://www.nieman.harvard.edu/reports/article/100503/ Teaching-Journalism-for-an-Unknown-Future.aspx $>$ [27-10-13]

GARCÍA, E.; DE LA MORENA, M.; MELENDO, L. (2012). “Análisis del valor comunicativo de las redes sociales en el ámbito universitario: Estudio de los usos de Twitter en el aula". En: Estudios sobre el Mensaje Periodístico, vol. 18, núm. especial octubre, p. 393-403.

GÓMEZ, M.; ROSES, S.; FARIAS, P. (2012). "El uso académico de las redes sociales en universitarios". En: Comunicar, vol.19, n. ${ }^{\circ} 38$, p. 131-138.

HIRST, M.; TREADWELL, G. (2011). "Blogs bother me". En: Journalism Practice, vol. 5 , n. ${ }^{\circ} 4$, p. $446-461$.

LYNCH, D. (2007). "Incubating innovstion at Journalism schools". En: Nieman Reports, vol. 61, n. ${ }^{0} 3 .<$ http://www.nieman.harvard.edu/reports/article/100167/ Incubating-Innovation-at-Journalism-Schools.aspx $>$ [28-10-13]

MICÓ, L.L.; MASIP, P.; BALLANO, S. (2012). "Criterios de contratación y perfiles profesionales emergentes en los medios. Universidad y empresas informativas en época de crisis en Cataluña". En: Ámbitos, n. ${ }^{\circ}$ 21, p. 281-294.

MOLINA, P. (2008). "Ciberperiodismo e interactividad: entre la participación y la apariencia". En: Anàlisi, n. ${ }^{\circ}$ 36, p. 175-182. 
TEJEDOR, S. (2008). "La enseñanza del ciberperiodismo en las materias de producción periodística de las licenciaturas de Periodismo". En: Estudios sobre el Mensaje Periodístico, vol. 14, p. 617-630.

LASSILA-MERISALO, M.; USKALI, T. (2011). "How to educate innovation journalists? Experiences of innovation journalism education in Finland 2004-2010". En: Journalism \& Mass Communication Educator, vol. 66, n. ${ }^{\circ} 1$, p. 25-38.

MANDEL, M. (2013). "Declining industries vs growing Jobs: what the WaPo deal tells us about innovation". En: The Progressive Fix. <http://www.progressivepolicy.org/2013/08/what-the-washington-post-deal-tells-us-about-the-future-ofjobs/>. [28-10-13]

NÚÑEZ GÓMEZ, P.; TEIXEIRA, S.; GARCÍA GUARDIA, M.L. (2012). "La profesión en el aula. Cómo investigar y mejorar el trabajo del futuro. El caso del Community Manager: diseño e investigación en TIC". En: Estudios sobre el mensaje periodístico, vol. 18, núm. especial noviembre, p. 951-957.

PEARCE, N.; WELLER, M.; SCANLON, E.; ASHLEIGH, M. (2010). "Digital scholarship considered: How new technologies could transform acadmeic work". En: In Education, vol. 16, n. ${ }^{\circ} 1 .<$ http://www.ineducation.ca/index.php/ineducation/article/view/44/508 > [27-10-13]

PRENSKY, M. (2001). "Digital Natives, Digital Immigrants". En: On the Horizon, vol. 9 , n. ${ }^{\circ}$, p. 1-6.

SÁNCHEZ, H.M.; MÉNDEZ, S. (2013). “Perfiles profesionales 2.0? Una aproximación a la correlación entre la demanda laboral y la formación universitaria". En: Estudios sobre el mensaje periodístico, vol. 19, núm. especial abril, p. 981-993.

WENGER, D.H.; OWENS, L.C. (2012). "Help Wanted 2010: An examination of new media skills required by top U.S. news companies". En: Journalism \& Mass Communication Educator, vol. 67, n. ${ }^{\circ}$, p. p-25.

\section{Las autoras}

Bella Palomo. Profesora titular en el departamento de Periodismo de la UMA, coordinadora del grado e investigadora principal del proyecto nacional "Audiencias Activas y Periodismo: Estrategias de Innovación para la Empresa Informativa y Nuevas Figuras Profesionales", ha sido profesora visitante en las Universidades de Washington (2004), Rutgers (2005) y Salvador de Bahía (2009), y consultora en la UOC. Sus investigaciones se han centrado en el estudio de Internet, la adaptación del periodista al entorno digital, el diseño editorial, los medios sociales y la innovación educativa.

María Sánchez González. Doctora en Periodismo por la Universidad de Málaga (2007), actualmente desarrolla su labor en el ámbito de la innovación, la comunicación y educación en red y los denominados social media, desde dos universidades públicas andaluzas. En la Universidad de Málaga actualmente es investigadora del proyecto "Audiencias Activas y Periodismo: Estrategias de Innovación para la 
Empresa Informativa y Nuevas Figuras Profesionales" e imparte docencia, como profesora asociada, en Periodismo, desde 2009, labores que compagina con su actividad como técnico de Innovación en la Universidad Internacional de Andalucía. 